\title{
Modelagem Digital de Dados Geológicos do Afloramento Morro Papaléo, com Base na Integração de Dados de (Sub) superfície
}

Reginaldo Macedônio da Silva', Maurício Roberto Veronez'2, Sérgio Florêncio de Souza', ${ }^{1}$ IGEO/DEG/UFRGS, ${ }^{2}$ UNISINOS

Copyright 2021, SBGf - Sociedade Brasileira de Geofísica

This paper was prepared for presentation during the $17^{\text {th }}$ International Congress of the Brazilian Geophysical Society held in Rio de Janeiro, Brazil, 16-19 August 2021.

Contents of this paper were reviewed by the Technical Committee of the $17^{\text {th }}$ International Congress of the Brazilian Geophysical Society and do not necessarily represent any position of the SBGf, its officers or members. Electronic reproduction or storage of any part of this paper for commercial purposes without the written consent of the Brazilian Geophysical Society is prohibited.

\section{Abstract}

Digital modeling of geological data is an important resource, which can contribute to the interpretation of architectural elements in different types of depositional systems. With the use of new technologies such as Terrestrial Laser Scanner and GPR, the challenge is to integrate external and internal data from an outcrop, to work on a threedimensional model, georeferenced by GNSS. For this analysis, the Morro Papaléo Outcrop was used as the study area, with characteristics of fluvial depositional processes, located in the municipality of Mariana Pimentel. In this work it was possible to integrate several data sources, obtained with different tools, which allowed the generation of geological surfaces, based on the interpretation of the point cloud and on the geophysical sections. It was also possible to integrate elements obtained with the use of Total Station, based on points georeferenced with GNSS. During the integration of the data it was possible to evaluate the positional accuracy of the topography of the terrain in the subsurface data, obtained with GPR.

\section{Introdução}

Existem diversas técnicas para analisar afloramentos, porém, com o surgimento de novas tecnologias em sensoriamento remoto, possibilitou um grande avanço na área da Geologia com a utilização de Laser Scanner Terrestre (LST) e a sua possível integração com dados geofísicos, entre outros.

Pode-se observar o avanço do LST, quando comparado com os métodos tradicionais, utilizados pelos geólogos em trabalhos de campo, como por exemplo o uso de bússola, fotografias digitais para montagem de fotomosaico e medidas de espessuras de camadas com o uso de trena. O LST é uma evolução quando comparado com outras tecnologias, como a medição com estação total e GNSS (Global Navigation Satelitte System), que são utilizados para obtenção de medidas e posicionamento de pontos georreferenciados. Outra informação que pode ser explorada pelo LST, é a obtenção da intensidade do sinal emitido pelo equipamento, podendo-se utilizar esse dado em outras análises como classificação de rochas em afloramentos (Kurz et al., 2008).

No Brasil, a aplicação de Laser Scanner na Modelagem Digital de Afloramentos (MDA) está em fase de desenvolvimento, e existem muitas análises a serem estudadas e comparadas com os métodos tradicionais para verificar o ganho de tempo e a qualidade na obtenção dos dados que essa ferramenta pode propiciar.

Modelos digitais tridimensionais possuem aplicações diversas e sua utilização vem crescendo no cenário mundial, em especial aqueles obtidos a partir de Laser Scanner Terrestre (Abmayr et al., 2004; Bellian, et al., 2005; Buckley et al., 2006). No Brasil, ainda são poucos os estudos que discutem as vantagens, os métodos de trabalho e as aplicações desta ferramenta (Freire, 2006; Barchik et al., 2007; Wutke \& Centeno, 2007; Teixeira, 2008; Centeno et al., 2010; Jacobi et al., 2010; Ferrari et al., 2012). Alguns trabalhos exploram também a intensidade do sinal para classificação de rochas (Inocencio et al., 2014) e aplicações em ciências florestais (Bordin et al., 2013).

Uma técnica que evoluiu rapidamente foi 0 georreferenciamento de informações geológicas por meio de Sistemas Globais de Navegação por Satélite - GNSS. Tais sistemas vêm permitindo ganhos tanto em exatidão quanto em tempo, quando da integração de diferentes produtos geológicos em um único sistema de referência, garantindo uma maior integridade nos processos de geração de modelos geológicos tridimensionais (Pringle et al., 2004; Thurmond et al., 2005; White \& Jones, 2008).

Em alguns trabalhos, como por exemplo Teixeira (2008), observou-se que o sistema LST permite uma análise da superfície do afloramento, que pode ser modelada através de ferramentas, como o uso do software GOCAD (Geological Object Computer Aided Draw). Porém, este modelo fica restrito à superfície externa do afloramento, visto que o Laser Scanner não atinge as partes internas do mesmo, sendo necessário o uso de outras tecnologias como o GPR (Ground Penetration Radar - Radar de Penetração) para complementação do modelo, pois com esta tecnologia é possível analisar subsuperfícies, que podem ser interpretadas e modeladas em conjunto com os dados do Laser Scanner Terrestre.

Segundo Bristow \& Jol (2003), o uso de GPR em estudos de depósitos de ambientes fluviais, para identificação de deposição em grande escala, como barra em pontal e outros elementos arquiteturais, tem sido muito utilizado para estudos de subsuperfície e que podem ser integrados com dados de superfície.

Para modelagem geológica, a tecnologia mais adequada para capturar dados de um afloramento depende de 
vários fatores, dentre eles, o objeto de estudo, a natureza do afloramento, incluindo quantidade de exposição e acessibilidade, nível de detalhe e precisão espacial necessária e as restrições que envolvem tempo e custo (Jones et al., 2008).

A modelagem geológica é utilizada como um meio de compreender arquiteturas geológicas e os seus efeitos sobre o comportamento em análogos de rochasreservatório (Kurz et al., 2008), além de permitir reunir em um mesmo modelo, informações de topologia, geometria, propriedades e atributos de objetos geológicos.

A utilização de afloramentos análogos na caracterização de atributos físicos de corpos sedimentares e na definição de modelos geológicos é um procedimento de caráter preditivo, devendo ser confiável, permitindo acessar e quantificar os atributos arquiteturais e geométricos dos estratos sedimentares (Paim et al., 2003). Assim, estas novas tecnologias podem permitir esta caracterização, pois subsidiam a interpretações de informações geológicas com uma visão tridimensional (3D) e com precisão submétrica na medição dos elementos.

Dentro desse contexto, para o desenvolvimento do trabalho foi necessária uma análise da exatidão posicional da nuvem de pontos. Com essa análise foi possível agregar, a nuvem de pontos, os dados de subsuperfície obtidos com GPR, para posterior interpretação dos elementos arquiteturais no modelo tridimensional gerado.

\section{Metodologia}

A área de estudo é um afloramento da Formação Rio Bonito, Permiano Inferior da Bacia do Paraná, chamado Morro Papaléo localizado no município de Mariana Pimentel (Figura 1), entre as coordenadas geodésicas: latitudes $30^{\circ} 18^{\prime} 10^{\prime \prime} \mathrm{S}$ e $30^{\circ} 18^{\prime} 40^{\prime \prime} \mathrm{S}$ e longitudes $51^{\circ} 28^{\prime} 20^{\prime \prime} \mathrm{W}$ e $51^{\circ} 38^{\prime} 20^{\prime \prime} \mathrm{W}$ no datum SIRGAS2000. A área mencionada está localizada em uma pedreira abandonada, originalmente utilizada na exploração de caulim. O afloramento tem uma visão tridimensional com uma boa exposição de rochas, como siltito fossilífero, siltito carbonoso, diamictito e arenito.

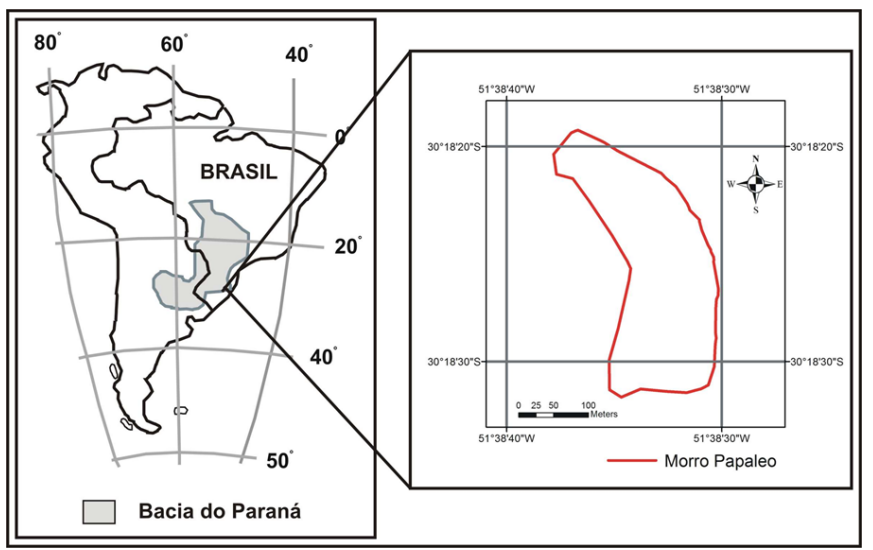

Figura 1 - Área de estudo
Para obtenção dos dados de superfície, foram seguidas as etapas definidas no fluxograma 1 apresentado na Figura 2.

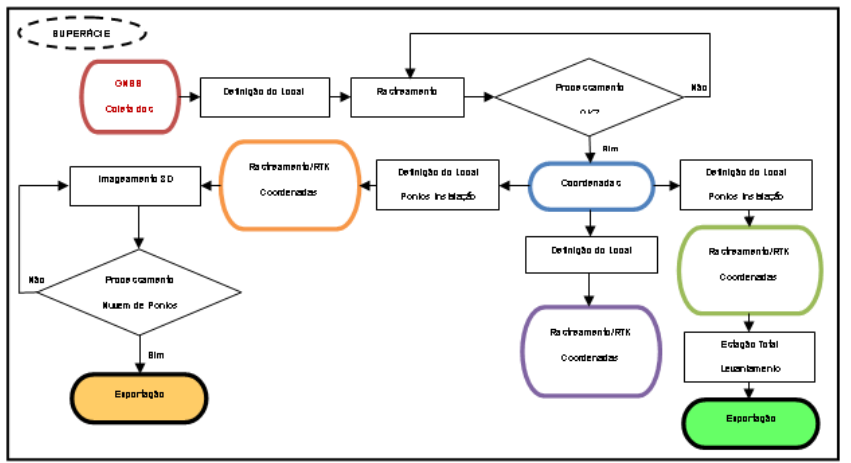

Figura 2 - Fluxograma 1 - Superfície

Com as coordenadas obtidas nos dados de superfície, estas foram utilizadas em três situações no trabalho de campo:

- Transporte de coordenadas para os pontos de instalação da estação total;

- Transporte de coordenadas para os pontos de instalação do laser scanner;

- Transporte de coordenadas para os pontos que definiram as linhas (seções) GPR.

Para o imageamento do afloramento Morro Papaléo foi necessário a instalação do GNSS-RTK (Real-Time Kinematic) no marco geodésico (ponto base), no qual executou-se o transporte de coordenadas aos pontos de controle (para uso do LST).

Foi necessário implantar 8 (oito) pontos de controle, para o imageamento, sendo 4 (quatro) pontos na parte leste do afloramento e os outros 4 (quatro) pontos na parte oeste.

É importante ressaltar que, no trabalho de campo, devese garantir uma sobreposição mínima de $10 \%$ entre as cenas imageadas, para que ocorra uma junção adequada, e também, para minimizar os efeitos provocados pela formação de sombras durante o imageamento (Bellian et al., 2005), que ocorrem devido a obstáculos encontrados no campo, como vegetações, orientação e variações do relevo da superfície a ser escaneada.

Durante o trabalho de campo, o LST foi instalado em pontos estratégicos, possibilitando que eles fossem intervisíveis e permitisse a determinação do azimute do escaneamento, utilizando-se um dos pontos de controle como ponto de ré. Este procedimento teve a duração de aproximadamente 8 horas.

Para o processamento dos dados do laser scanner terrestre, seguiu-se os procedimentos da Figura 3: 


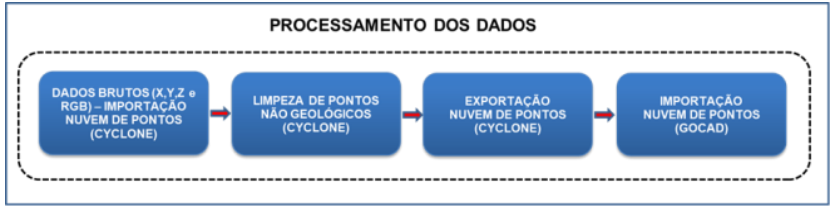

Figura 3 - Processamento nuvem de pontos

Com os dados importados, foi necessário executar a limpeza de pontos não geológicos, como vegetação e pilhas de materiais soltos no entorno do afloramento, para facilitar a visualização das camadas (Figura 4A e 4B).

$\mathrm{Na}$ Figura 4C é possível observar o resultado da triangulação da nuvem de pontos, após a importação advinda do software Cyclone.

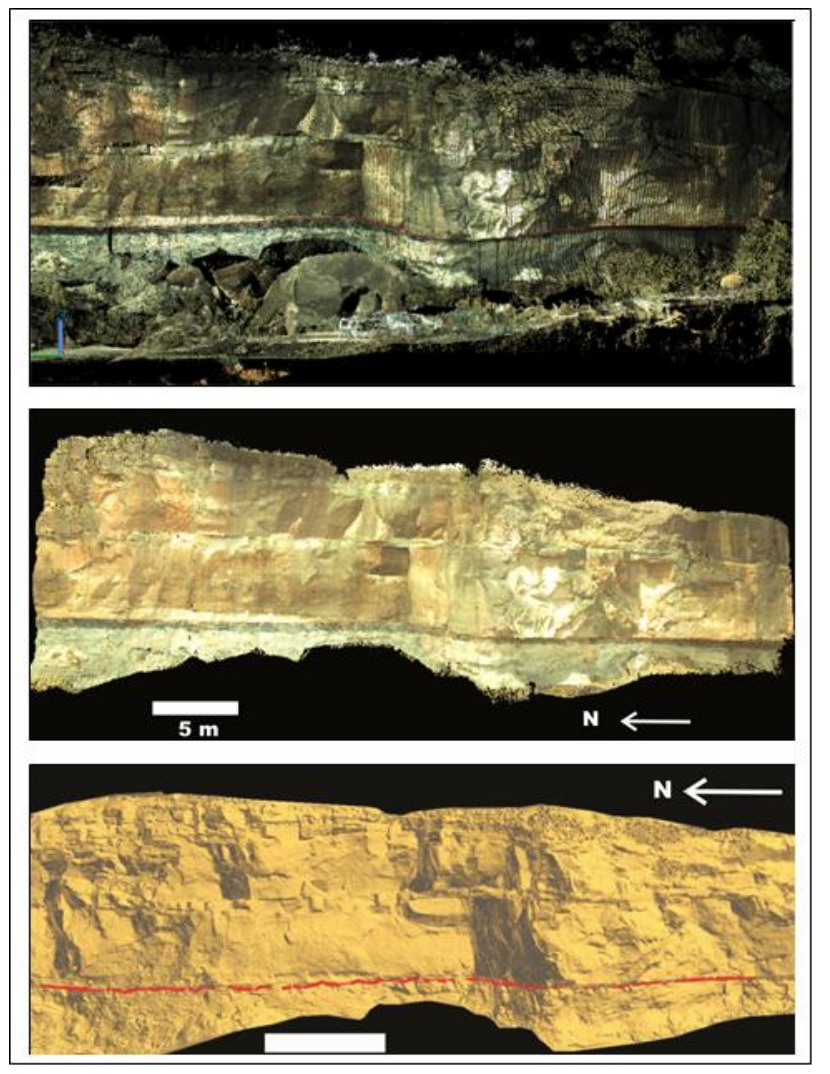

Figura 4 (A, B e $\boldsymbol{C})$ - (4A) Nuvem de pontos sem a limpeza de obstruções - (4B) Nuvem de pontos com a limpeza das obstruções - (4C) Triangulação executada no software GOCAD, com uma linha inserida a partir da medição de campo com estação total.

Para o levantamento dos dados de subsuperfície, de acordo com o fluxograma 2 da Figura 5, foi necessário executar a aquisição das informações por meio de equipamento GPR, com base nas linhas locadas na parte superior do afloramento.

No trabalho de campo, foram levantadas as seções geofísicas longitudinais (5 - cinco) e transversais (8 oito), num total de 13 (treze) seções, cujas maiores tinham 300 (trezentos) metros de comprimento, e para isso, utilizou-se uma antena de $100 \mathrm{MHz}$.

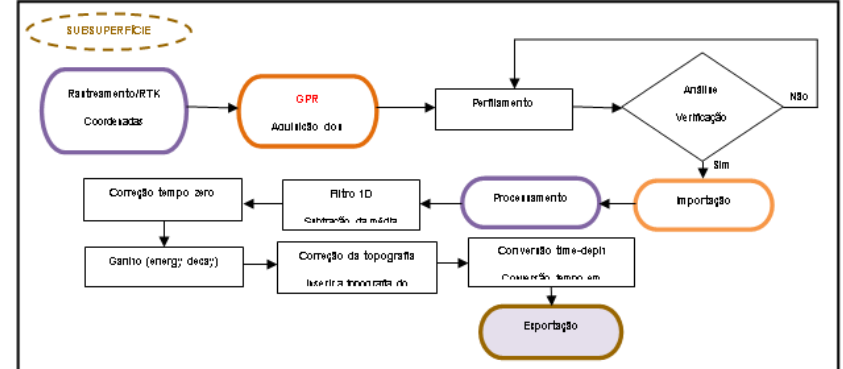

Figura 5- Fluxograma 2 - Subsuperfície

Para inserir a topografia em cada seção geofísica foi necessário criar um arquivo txt com as coordenadas ( $X$, $Y, Z$ ) de cada perfil (alinhamento da seção), com base nos dados da topografia do terreno levantada com Estação Total. Deve-se observar que para 0 levantamento topográfico planialtimétrico do topo do afloramento foi utilizado o Datum SIRGAS2000, como sistema de referência para as coordenadas planas na Projeção UTM (Universal Transversa de Mercator), o mesmo sistema utilizado no imageamento com Laser Scanner Terrestre, obtendo-se desta forma um referencial único para os dados de campo.

Finalizada a correção da topografia do terreno, executouse a migração do tempo em profundidade, no qual utilizou-se como velocidade média de profundidade 0,10 m/ns (obtida na seção geofísica 15-13).

Concluída a conversão do tempo em profundidade, exportaram-se todos os radargramas, com o uso do software Reflexw, utilizando-se como extensão o formato SEGY (Society of Exploration Geophysicists $Y$ format), sendo este, utilizado na importação de dados geofísicos no software de modelagem GOCAD.

\section{Resultados}

Com os resultados dos dados de superfície e subsuperfície foi executada a modelagem dos elementos interpretados, conforme informações do fluxograma 3 da Figura 6. Para isso se utilizou a nuvem de pontos do laser scanner terrestre (LST), as coordenadas obtidas da estação total (ET) e os radargramas (seções geofísicas GPR). Os elementos interpretados foram utilizados na caracterização das feições geológicas do afloramento, para obtenção de um modelo tridimensional.

No processamento da exportação da nuvem de pontos para o software GOCAD, foi necessário exportar os dados no formato TXT com as coordenadas X, Y, Z, e neste caso, não se exportou as informações RGB (Red Green Blue), advindas do equipamento LST, pois no software GOCAD não permitia a importação dessa informação. Porém, em algumas etapas da interpretação foi necessário trabalhar no software Cyclone, pela facilidade de manipulação da nuvem de pontos, que permitiu a vetorização do limite entre as fácies do afloramento. 


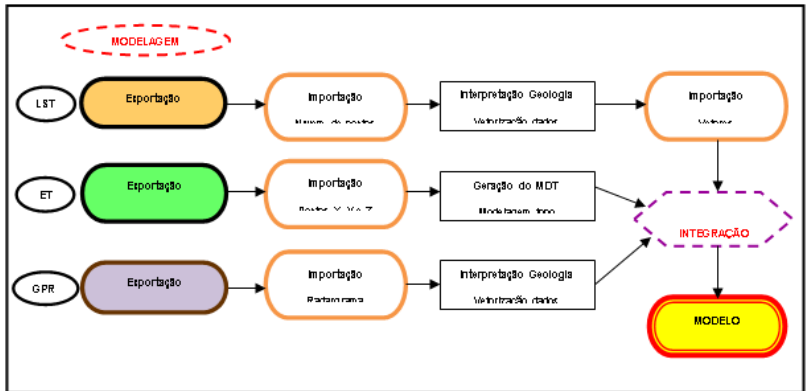

Figura 6 - Fluxograma 3 - Modelagem

Com a nuvem de pontos importada, realizou-se uma análise do georreferenciamento, usando-se como parâmetro os dados coletados em campo com estação total, apoiados nos pontos georreferenciados com GNSS. Para isso foi realizado, no campo, a medição da camada de carvão em sua parte superior, que posteriormente, adotou-se como referência na análise do posicionamento. Este procedimento foi necessário, para verificar se o georreferenciamento manteve-se correto após a importação no software GOCAD, pois as informações obtidas foram integradas posteriormente, com dados de subsuperfície.

Um dos elementos geológicos utilizado na interpretação do modelo foi a camada de carvão, pois esta superfície estava presente nas duas faces (leste e oeste) do afloramento, o que permitiu utilizá-la como datum de referência para as outras camadas interpretadas.

Deve-se ressaltar que o resultado da análise da exatidão posicional do georreferenciamento da nuvem de pontos, constituiu uma importante etapa da modelagem, pois assim, manteve-se uma padronização no posicionamento da nuvem de pontos. Com as informações validadas, efetuou-se a triangulação da nuvem de pontos, e posteriormente, a limpeza das triangulações incorretas, e assim, obteve-se os dados do laser scanner terrestre, processado e triangulado, que permitiu a modelagem do carvão entre os dois lados do afloramento e a integração com as seções geofísicas (Figura 7).

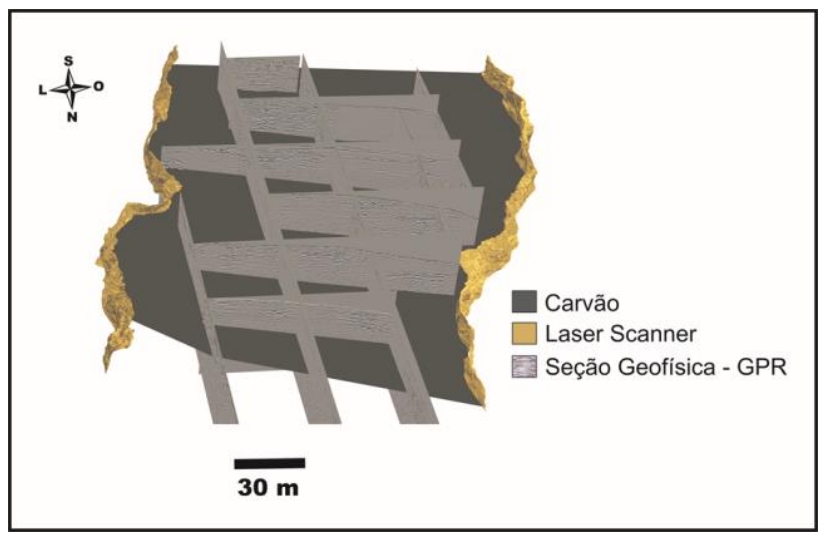

Figura 7- Modelagem da camada de carvão

\section{Conclusão}

Pode-se concluir que a metodologia utilizada para a integração dos dados de superfície com o uso de Laser Scanner Terrestre e os dados de subsuperfície com o uso de GPR foi adequada para a modelagem digital de afloramento, na qual foi possível integrar, também, dados advindos de outras técnicas e equipamentos como Estação Total e GNSS, por meio da utilização de um referencial único para todo o levantamento de campo.

Para cada etapa de aquisição dos dados no campo, tevese a preocupação de avaliar o posicionamento dos elementos, referente às coordenadas utilizadas em todo o processo. Isso foi avaliado com a obtenção de coordenadas obtidas ao longo do afloramento medido com a Estação Total e amarradas em pontos georreferenciados a partir do marco geodésico implantado no topo do afloramento. Esta análise foi executada tanto para os dados de superfície quanto para os dados de subsuperfície.

A manipulação dos dados foi executada em diversos softwares, o que permitiu, em alguns casos, obter informações das camadas do afloramento com mais detalhes, visto que uma única ferramenta não permite a interpretação de todos os dados vindos de fontes diferentes.

Quanto à camada de carvão, nas seções geofísicas foi possível identificá-la em três locais diferentes, sendo estas, integradas também, com a interpretação da nuvem de pontos e com o levantamento de campo executado com estação total, visto que uma única técnica não conseguiria coletar toda a camada devido a particularidade do afloramento. O procedimento utilizado neste trabalho poderá ser aplicado em outros afloramentos que necessitem ser mapeados num referencial tridimensional.

\section{Agradecimentos}

Agradeço a CAPES/PROSUP pela bolsa concedida, pois este apoio foi fundamental para que eu pudesse trabalhar nesse novo desafio.

À PETROBRAS pelo apoio financeiro aos projetos NEAP (Núcleo de Estratigrafia Aplicada, Convênio 16 - SAP 4600242459) e "Mapeamento 3D Georreferenciado de Afloramentos Utilizando uma Técnica LIDAR (Light Detection And Ranging)", (Termo de Cooperação 0050 0044869. 08.4 - SAP: 4600285973), ambos financiados pela Rede Tecnológica em Sedimentologia e Estratigrafia.

\section{Referências}

Abmayr, T., Härtl, F.; Mettenleiter, M., Heinz, I., Hildebrand, A., Beumann, B., Fröhlich, C. 2004. Realistic 3D-Reconstruction - Combining Laserscan Data with RGB-Color Information. ISPRS-International Archives of Photogrammetry, Remote Sensing and Spatial Information Sciences. Vol XXXV, Part B5: 198-203. 
Barchik, E., Moser, I., Santos, D. S. dos, Martins, B. D. 2007. Aplicação do Scanner Terrestre ILRIS - 3D no Ramo da Mineração. Anais. XIII Simpósio Brasileiro de Sensoriamento Remoto, Florianópolis, Brasil, 21-26 abril 2007, INPE, p. 3631-363

Bellian, J. A., Kerans, C., Jennette, D.C. 2005. Digital outcrop models: applications of terrestrial scanning lidar technology in stratigraphic modeling. Journal of Sedimentary Research, n. 75, p.166-176.

Bordin, F.; Teixeira, E. C.; Rolim, S. B. A.; Tognoli, F. M. W.; Souza, C. N.; Veronez, M. R. 2013. Analysis of the influence of distance on data acquisition intensity forestry targets by a lidar technique with terrestrial laser scanner. The international archives of the photogrammetry, remote sensing and spatial information sciences (cd-rom), v. XI2/w1, p. 99-103, 2013.

Bristow, C. S. \& Jol, H. M. 2003. An introduction to ground penetrating radar (GPR) in sediments. Geological Society, London, Special Publications 2003; v. 211; p. 1-7 doi: 10.1144/GSL.SP.2001.211.01.01.

Buckley, S. J., Howell, J.A., Enge, H.D., Leren, B.L.S. and Kurz, T.H. 2006. Integration of terrestrial laser scanning, digital photogrammetry and geostatistical methods for high-resolution modelling of geological outcrops. In: The International Archives of the Photogrammetry, Remote Sensing and Spatial Information Sciences. Dresden, Germany, Vol. XXXVI, Part B5, proceedings CD.

Centeno, J. A. S., Wutke, J. D., Mitishita, E. A., Vögtle, T. 2010. Two Methods to Estimate the Spot Size of Terrestrial Laser Scanners. Journal of Surveying Engineering, v. 136, p. 126-131.

Ferrari, F., Veronez, M. R., Tognoli, F. M. W., Inocêncio, L. C., Paim, P. S. G., Silva, R. M. da. 2012. Visualização e Interpretação de Modelos Digitais de Afloramentos Utilizando Laser Scanner Terrestre. Geociências (São Paulo. Online), v. 31, p. 79-91.

Freire, R. C. 2006. Técnicas de aquisição de dados geológicos com a tecnologia LIDAR. Dissertação (Mestrado em Geodinâmica e Geofísica) - Universidade Federal do Rio Grande do Norte, 54p.

Inocencio, L. C., Veronez, M. R., Tognoli, F. M. W., Souza, M. K., Silva, R. M., Gonzaga JR, L., Silveira, C. L. B. 2014. Spectral Pattern Classification in Lidar Data for Rock Identification in Outcrops. The Scientific World Journal, v. 2014, p. 1-10, 2014.
Jacobi. L., Silva, R. M. da, Markoski, P.R., Veronez, M.R., Tognoli, F.M.W., Paim, P.S.G., Faccini, U.F., Lavina, E.L.C. 2010. Modelo Digital de Afloramento visando a análise e interpretação geológica: um estudo de caso baseado em dados de Laser Scanner 3D Terrestre, Anais do $45^{\circ}$ Congresso Brasileiro de Geologia PAP916.pdf.

Jones, R. R., Mccaffrey, K. J. W., Imber, J., Wightman, R., Smith, S. A. F., Holdsworth, R.E., Clegg, P.; Paola, N. de, Healy, D., Wilson, R.W. 2008. Calibration and validation of reservoir models: the importance of high resolution, quantitative outcrop analogues, Geological Society, London, Special Publications, January 1, 2008; 309(1): 87-98.

Kurz, T. H., Buckley, S. J., Howell, J. A., Schneider, D. 2008. Geological Outcrop Modelling and Interpretation Using Ground Based Hyperspectral and Laser Scanning Data Fusion. International Archives of the Photogrammetry, Remote Sensing and Spatial Information Sciences, 37(B8): 1229-1234, 2008. Disponível em http://www.isprs.org/proceedings/XXXVII/congress/8_pdf/ 12_WG-VIII-12/09.pdf Acesso em 27 de fevereiro de 2009.

Paim, P. S. G., Faccini, U. F. \& Netto, R. G., 2003 Geometria, arquitetura e heterogeneidades de corpos sedimentares - Estudo de Casos. In: Paim, P. S. G., Garcia, A. J. V., Faccini, U. F. \& Lavina, E. L. C. (eds.) GEOARQ: Uma Abordagem Integrada e Aplicada da Geologia Sedimentar. UNISINOS. Programa de PósGraduação em Geologia, p.:15-25.

Pringle, J. K., Westerman, A. R., Clark, J. D., Drinkwater, N. J., Gardiner, A. R. 2004. 3D high-resolution digital models of outcrop analogue study sites to constrain reservoir model uncertainty: an example from Alport Castles, Derbyshire, UK. Petroleum Geoscience, 10, 343-352.

Teixeira, W. L. E. 2008. Aquisição e construção de modelos estáticos análogos a reservatórios petrolíferos com tecnologia LIDAR e GEORADAR. Dissertação de Mestrado, Universidade Federal do Rio Grande do Norte. Natal, 99p.

Thurmond, J. B., Drzewiecki, P. A., XU, X. 2005. Building simple multiscale visualizations of outcrop geology using virtual reality modeling language (VRML). Computers and Geosciences, 31, 913-919.

White, P. D. \& Jones, R. R. 2008. A cost-efficient solution to true color terrestrial laser scanning, Geosphere, June 1, 2008, 4(3): 564 - 575. 
Wutke, J. D., Centeno, J.S. 2007. Métodos para avaliação da resolução de sistemas de varredura a laser terrestres. Boletim de Ciências Geodésicas, v. 13, p. 151164. 